hep-ph/9406365

\title{
Quark-Gluon Plasma Freeze-Out from a Supercooled State?
}

\author{
T. Csörgö ${ }^{1}$ 周 and L. P. Csernai ${ }^{2}$ \\ ${ }^{1}$ KFKI Research Institute for Particle and Nuclear Physics of the \\ Hungarian Academy of Sciences, H-1525 Budapest 114, P.O. Box 49. Hungary \\ ${ }^{2}$ Center for Theoretical Physics, Physics Department, \\ University of Bergen, Allegaten 55, N-5007 Bergen, Norway
}

\begin{abstract}
The quark-gluon plasma, formed in the first $3 \mathrm{fm} / \mathrm{c}$ of the heavy ion collisions at RHIC and LHC, supercooles due to nucleation and develops soon a negative pressure in the bag model. The negative pressure yields mechanical instability which may lead to a sudden timelike deflagration to a (super)heated hadron gas. The resulting freeze-out times are shorter than those of the standard nucleation scenario.
\end{abstract}

Accepted for publication in Physics Letters B.

*E-mails: csorgo@sunserv.kfki.hu

csernai@sentef1.fi.uib.no 
Large experimental and theoretical programmes were launched for studying the properties of Quantum Chromo Dynamics (QCD) at high temperatures and energy densities [1]. At BNL reactions of $A u$ nuclei with $200 \mathrm{AGeV}$ cms energy are expected to create a hot blob of gluons and quarks, while $\mathrm{Pb}$ nuclei are to be collided at the CERN LHC with $6.3 \mathrm{ATeV}$ energy in the c.m. frame. The nuclei pass through each other at these high energies, leaving behind a highly excited volume filled with gluons and quarks [2].

Supercooling, entropy production and bubble kinetics in the Quark-Gluon Plasma were already studied 10 years ago, see e.g. ref. [3]. However, at that time no dedicated nucleation calculations were preformed, e.g. the dynamical pre-factor [4] was not known and the effects coming from the admixture of hadronic bubbles to the supercooled QGP were neglected [3]. The phenomenology of the high energy heavy ion collisions in the RHIC and LHC energy region was not known and some of the calculations were preformed [3] in the context of the hadronization of the early universe.

The dynamics of the rehadronization of the expanding and cooling plasma phase is very sensitive to the formation rate of hadronic bubbles inside the plasma. In the thermally overdamped limit the characteristic nucleation time was found to be of the order of 100 $\mathrm{fm} / \mathrm{c}$ for a longitudinally expanding gas of gluons and massless quarks rehadronizing into a massless pion gas [4]. Let's recite these results which we need for our considerations about the time-scales of the ultra-relativistic heavy ion collisions. The plasma will cool according to the law $T(t)=T_{0}\left(t_{0} / t\right)^{1 / 3}$ until $t_{c} \approx 3 \mathrm{fm} / \mathrm{c}$. The matter continues to cool below $T_{c}$ until to about $0.95 T_{c}$ when noticeable nucleation begins. When the temperature has fallen to a "bottom" temperature, $T_{b} \approx 0.8 T_{c}$, bubble formation and growth is sufficient to begin the reheating the system at about $t_{b} \approx 7 \mathrm{fm} / \mathrm{c}$. When the temperature exceeds about $0.95 T_{c}$ nucleation of new bubbles shuts off. The transition continues only because of the growth of previously created bubbles. Compared to the idealized adiabatic Maxwell-Boltzmann construction which assumes phase equilibrium at $T_{c}$ the finite transition rate delays the completion of the transition by $\approx 11 \mathrm{fm} / \mathrm{c}$, yielding a completion time of $t_{c o m}=50 \mathrm{fm} / \mathrm{c}$. Detailed calculations including dilution factor for the bubble formation, spherical expansion, 
bubble fusion and varying the values for the surface tension do not change the qualitative behavior of the rehadronization process. According to the calculations in [5], the time-scales become somewhat shorter, due to the fusion of the bubbles.

Present experiments indicate early freeze-out: i) HBT results, ii) strange antibaryon enhancement, iii) high effective temperatures and iv) unchanged hadronic masses.

Detailed scan of the freeze-out surface is given by the side, out and longitudinal components of the Bose-Einstein correlation function (BECF) at various rapidities and transverse momenta [6]. The longitudinal radius, $R_{L}$, is proportional to the freeze-out proper-time, $t_{f}$, since the BECF measures only that piece of the longitudinally expanding tube, where the rapidity distributions belonging to different spatial rapidities overlap (thus pions with similar momenta emerge). The size of this region is characterized by $\Delta \eta$, the width of the rapidity distribution at a fixed value of the spatial rapidity [7]. For one dimensional expansion the length of the region with a given spatial rapidity width is just $t \Delta \eta$. The hydrodynamical formalism gave the result [8] $R_{L}=t_{f} \Delta \eta=t_{f} \sqrt{T_{f} / m_{T}}$, where $T_{f}$ is the freeze-out temperature and $m_{T}$ is the transverse mass of pions. This result was confirmed in a detailed three dimensional hydrodynamical simulation which included transverse flow, nontrivial freeze-out geometry and resonance contributions to the pion spectra [9]. The side component, $R_{T, \text { side }}$, measures the geometrical radius of the pion source at the freeze-out time, [G]. The out component, $R_{T, \text { out }}$ is sensitive also to the duration of the pion emission [7]. The BECF in terms of the momentum difference of the pair, $\mathbf{Q}$, is fitted with the form $C\left(Q_{T, \text { side }}, Q_{T, \text { out }}, Q_{L}\right)=1+\lambda \exp \left(-R_{T, \text { side }}^{2} Q_{T, \text { side }}^{2}-R_{T, \text { out }}^{2} Q_{T, \text { out }}^{2}-R_{L}^{2} Q_{L}^{2}\right)$. The intercept parameter, $\lambda$, stands for particle mis-identification, acceptance cuts and long-lived resonance effects.

The transverse radius parameter of BECF-s scales with the rapidity density for high energy reactions as $R_{L}=R_{T, \text { side }}=R_{T, \text { out }}=c\left(\frac{d n^{ \pm}}{d y}\right)^{1 / 3}$. This scaling was shown to be valid for the transverse radius independently of the type and energy of the reaction including UA1, AFS, E802, NA35 and NA44 data, and can be explained based on general freeze-out arguments [10]. The exponent (1/3) indicates that pions freeze out at a given critical density 
and that the longitudinal radius is proportional to the transverse one. Actually they turned out to be equal, within NA35 and NA44 errors. We use this trend in the data at presently available energies to estimate the freeze-out proper-time at RHIC and LHC energies with a conservative extrapolation. The proportionality constant, $c$, was determined to be 0.9 using the $C=1+\lambda \exp \left(-R^{2} Q^{2} / 2\right)$ convention for the transverse radius [1]. Thus for our earlier mentioned parametrization the constant of proportionality is decreased by $\sqrt{2}$.

The charged particle rapidity density is about 133 at midrapidity for central ${ }^{32} S+{ }^{238} U$ collisions at CERN SPS corresponding to $R_{L}=4.5 \pm 0.5 \mathrm{fm}$ and a freeze-out time of $t_{f}=4.5-6.5 \mathrm{fm} / \mathrm{c}$. In a first order equilibrium phase transition the system has to spend a long time in the mixed phase to release latent heat and decrease the initially high entropy density, resulting in a a large difference between the side and out radii, [7] and also resulting in a large value for the total freeze-out time. Given the $4.5-6.5 \mathrm{fm} / \mathrm{c}$ freeze-out time the duration of the particle emission can only be shorter than this value, implying that a strong first order equilibrium phase transition cannot happen at present CERN SPS reactions.

The charged particle rapidity density was shown to scale with the projectile mass number in case of symmetric collisions as $\frac{d n^{ \pm}}{d y}=0.9 A^{\alpha} \ln \left(\sqrt{s} / 2 m_{p}\right)$, where the exponent $\alpha$ was found to be in the region $1.1 \leq \alpha \leq 4 / 3[11]$. Combining these equations the target mass and energy dependence of the freeze-out time, $t_{f}$, is given as

$$
t_{f}=0.58 A^{\alpha / 3} \sqrt{m_{T} / T_{f}} \ln ^{1 / 3}\left(\sqrt{s} / 2 m_{p}\right) .
$$

For various high energy heavy ion reactions we estimate the freeze-out proper-time using a conservative $\alpha=1.3$. The number of pions with a given $m_{T}$ is exponentially falling, the relative number of pions with $m_{T} \geq 2 T_{f}$ is rather small, giving $\sqrt{m_{T} / T} \approx 1$. - 1.4.

i) Summarizing the above, trends in present HBT data imply freeze-out time of 6-10 fm/c at CERN SPS for $\mathrm{Pb}+\mathrm{Pb}, 8-13 \mathrm{fm} / \mathrm{c}$ at RHIC for $\mathrm{Au}+\mathrm{Au}$ and 11-16 fm/c at $\mathrm{LHC} \mathrm{Pb}+$ $\mathrm{Pb}$ collisions. At these times the system is close to the bottom of the temperature curve, in the deepest supercooled quark-gluon plasma (QGP) state, according to the calculations in [4. [5]. 
ii) The idea that QGP has to hadronize suddenly from a deeply supercooled state has the consequence that the strange particle composition and especially the production rate of strange antibaryons as suggested by [12, 13] could become a clean signature of the QGP formation at RHIC and LHC energies as well as at the present CERN SPS energy. The WA85 collaboration found large production rates of strange antibaryons at CERN SPS S + $\mathrm{W}$ interactions [13]. The ratio $\bar{\Xi}^{-} / \bar{\Lambda}$ was found to be more than three times greater than those obtained for $\mathrm{p}+\mathrm{p}$ by the AFS collaboration, (a four standard deviation effect). Previous, larger value was reproduced by a sudden rehadronization from QGP near equilibrium, assuming fixed strangeness abundance [12]. Sudden spacelike detonations and deflagrations from a supercooled baryon rich QGP were related to strangeness enhancement at CERN SPS energies in refs. [14,15].

iii) At lower energies the effective temperature for the protons (baryons) is larger than that of pions [16] after the freeze-out of a hot fireball where resonances (deltas) had been in thermal equilibrium, and decayed after the freeze-out. The effective slope of the baryons is about $10 \%$ lower, than the freeze-out temperature. As it was noted by the NA35 collaboration at QM'93 [1] all strange particle transverse mass spectra are well described by a single exponential corresponding to "temperatures" of about $210 \pm 20 \mathrm{MeV}$, which are difficult to understand in a conventional hadron gas picture. The latent heat during a sudden breakup might be released as kinetic energy of the hadrons [17] in a timelike deflagration (TD). This may happen since the pressures before and after the TD hypersurface are not necessarily equal, thus part of the latent heat may be converted to work. This is in qualitative agreement with the observation that the multi-strange antibaryons observed by the WA85 collaboration at transverse momenta above $1.2 \mathrm{GeV} / \mathrm{c}$ show an effective $T_{\text {slope }} \approx 230$ $\mathrm{MeV}$. Pions exhibit a more moderate slope parameter than strange baryons. This can be attributed to the decays of baryonic resonances which produce dominantly low $p_{T}$ pions.

iv) In dense and hot matter hadronic masses are expected to decrease [1]. Nevertheless, in the dilepton spectra the observed masses of hadronic resonances (e.g. $\phi$ ) were identical to their free masses in heavy ion reactions at SPS energies. This can be attributed to 
simultaneous hadronization and freeze-out, where the medium effects are ceased to exist, when hadrons are formed.

Thus from trends in interferometry data the freeze-out time scale is short enough to prevent reheating and full rehadronization through bubble formation exclusively. This in turn implies that other mechanisms must dominate the final stages of the hadronization.

Dynamics of sudden freeze-out. Using a critical temperature $T_{c}=169 \mathrm{MeV}$ [ the pressure, $p$, of the supercooled QGP vanishes at $T=0.98 T_{c}$ already. The temperature of the system in the supercooled phase reaches $T=0.7-0.9 T_{c} \simeq 120-150 \mathrm{MeV}$ [5]. At such low temperatures the pressure of the QGP phase takes large negative values in the bag model. Systems with negative pressure are mechanically unstable, either they don't fill the available volume or they spontaneously cluster.

The mechanical instability of the QGP phase below $0.98 T_{c}$ and the typical $100 \mathrm{fm} / \mathrm{c}$ nucleation times are the basic reasons for the sudden rehadronization which we propose. The expansion in an ultra-relativistic heavy ion collision is so fast, that the temperature drops below $T_{c}$ by 20-30 \% before nucleation starts reheating the system. By that time the QGP is far in the mechanically unstable region. With the help of a timelike deflagration (TD) the system may jump from a mechanically unstable phase to a mechanically stable and thermodynamically (meta)stable phase, the (superheated) hadron gas state.

Let us consider the sudden freeze out from supercooled QGP 18, 19,14. Relativistic TD-s are governed [19] by conservation of the energy-momentum tensor and the entropy current across the front, expressed by the Taub adiabat,

$$
\frac{p_{1}-p_{0}}{X_{1}-X_{0}}=\frac{\omega_{1} X_{1}-\omega_{0} X_{0}}{X_{1}^{2}-X_{0}^{2}}
$$

the Rayleigh-line and the Poisson-adiabat,

$$
\frac{p_{1}-p_{0}}{X_{1}-X_{0}}=\omega_{0} \quad \text { and } \quad \frac{s_{1}^{2}}{\omega_{1}}=\frac{R^{2}}{X_{1}} \frac{s_{0}^{2}}{\omega_{0}}
$$

Here $\omega_{i}=\varepsilon_{i}+p_{i}$ denotes the enthalpy density, the quantity $X_{i}$ is defined as $X_{i}=$ $\omega_{i}\left(U_{i}^{\nu} \Lambda_{\nu}\right)^{2} / \omega_{0}\left(U_{0}^{\nu} \Lambda_{\nu}\right)^{2}$ the entropy density is denoted by $s_{i}$, the four-velocity relative to 
the deflagration hypersurface (characterized by the timelike normal vector $\Lambda^{\nu}$ ) is given by $U_{i}^{\nu}$. The index 0 refers to the quantity before the TD, while 1 refers to it after TD. If the flow follows a scaling Bjorken-expansion (either in one or in three dimensions) both before and after the TD, and the TD hypersurface is given by a constant proper-time, the above equations can be simplified. The Taub adiabat reduces to $\varepsilon_{1}=\varepsilon_{0}$, the Rayleigh-line becomes an identity and the Poisson-adiabat simplifies to the requirement that $R=s_{1} / s_{0} \geq 1$. If a TD starts from a $30 \%$ supercooled state the initial state is a mixture including $15-25 \%$ hadronic phase. The initial energy density in terms of the volume fraction of hadrons, $h$, is given by $\varepsilon_{0}\left(T_{0}\right)=h \varepsilon_{H}\left(T_{0}\right)+(1-h) \varepsilon_{Q}\left(T_{0}\right)$ and the expression for the entropy density is similar. In the bag model, the energy and entropy densities are given as $\varepsilon_{Q}=3 a_{Q} T_{Q}^{4}+B$, $\varepsilon_{H}=3 a_{H} T_{H}^{4}, s_{Q}=4 a_{Q} T_{Q}^{3}, s_{H}=4 a_{H} T_{H}^{3}$ with coefficients $a_{Q}=\left(16+21 n_{F} / 2\right) \pi^{2} / 90$ and $a_{H}=3 \pi^{2} / 90$. The bag constant is given by $B=\left(a_{Q}-a_{H}\right) T_{c}^{4}$. The quantity $r=a_{Q} / a_{H}$ gives the ratio of the degrees of freedom of the phases. Given the initial temperature and hadronic fraction by the nucleation scenario, the Taub and Poisson adiabats yield the entropy ratio and the temperature after the TD as

$$
\frac{T_{1}}{T_{c}}=\left[\frac{x-1}{3}+x \frac{T_{0}^{4}}{T_{c}^{4}}\right]^{1 / 4}, \quad R=\frac{1}{x}\left[\frac{T_{1}}{T_{0}}\right]^{3},
$$

where the ratio of the effective number of degrees of freedom is given by $x=h+r(1-h)$. These equations provide a range for possible values of $T_{H}=T_{1}$ and $T_{Q}=T_{0}$ for a given initial hadronic fraction, $h$, given by

$$
\begin{aligned}
{\left[\frac{x-1}{3}\right]^{1 / 4} } & \leq \frac{T_{H}}{T_{C}} \leq\left[\frac{x-1}{3\left(1-x^{-1 / 3}\right)}\right]^{1 / 4}, \\
0 & \leq \frac{T_{Q}}{T_{C}} \leq\left[\frac{x-1}{3\left(x^{4 / 3}-x\right)}\right]^{1 / 4} .
\end{aligned}
$$

The largest possible values for the temperature of the hadronic phase as well as the minimum supercooling in the initial phase-mixture corresponds to adiabatic $(R=1)$ TD-s. Entropy production in the transition decreases both the final and the initial temperatures at a given $h$. 
For large initial hadronic fraction, $h \geq 0.9$, TD-s become possible to final frozen-out hadronic states.

The sudden hadronization may easily [14] but not necessarily end up with a superheated hadronic gas, which is not frozen out yet. The freeze-out time $t_{f}$ can be calculated in a $d$ dimensional scaling Bjorken expansion model ( $d=1$ or 3 ) with the bag equation of state and including the entropy production in the nucleation scenario as:

$$
\tau_{f}=r^{1 / d} t_{c}\left(\frac{T_{c}}{T_{f}}\right)^{3 / d}+\Delta t_{f}
$$

where $t_{c}$ is the proper-time when the critical temperature is first reached by the cooling $\mathrm{QGP}, T_{f}$ is the freeze-out temperature and $\Delta t_{f}$ denotes the increase of the freeze-out time due to the non-adiabaticity of the nucleation. Thus the freeze-out time may be decreased by:

- Decrease of $r$. The quark degrees of freedom equilibrate much slower than the gluons during the first $3 \mathrm{fm} / \mathrm{c}$ at RHIC or LHC energies [20]. A hot glue scenario is also proposed where a hot gluonic plasma develops from the pre-equilibrium parton collisions. By decreasing the effective number of flavours from $n_{F}=2$ to $n_{F}=0$ the ratio $r$ decreases from $37 / 3$ to $16 / 3$.

- Increase of $d$. The time when QGP first reaches the critical temperature is prescribed by the Parton Cascade Model (PCM) calculations for the first $3 \mathrm{fm} / \mathrm{c}$ [2] and the subsequent hydrodynamical calculations. If we start the hydrodynamical evolution at $t_{I}=3.5 \mathrm{fm} / \mathrm{c}$ with a temperature given by PCM as $T_{I}=1.6 T_{c}$, for a $d=1$ Bjorken expansion the critical temperature is reached by $t_{c}(1)=14.3 \mathrm{fm} / \mathrm{c}$ while for a $d=3$ expansion the critical temperature is reached by $t_{c}(3)=5.6 \mathrm{fm} / \mathrm{c}$. Changing the dimension of the expansion from the initial value of 1 to the maximum value of 3 further decreases the cooling time in the hadronic phase from the critical temperature to the freeze-out temperature, as expressed by the factor $\left(T_{c} / T_{f}\right)^{(3 / d)}$ in the previous equation.

- Decrease of $\Delta t_{f}$. The nucleation process is governed by the dissipation around the hadronic bubbles and leads to extra entropy production, thus a delay in the freeze-out 
time by $\Delta t_{f}$ compared to the adiabatic expansion. If the TD happens at an early stage of the reaction, the net entropy production can be decreased since TD-s are governed by the mechanical instability in QGP and by the balance equations across the front and not by dissipation. Thus an early TD may decrease $\Delta t_{f}$.

Converting part of the latent heat to kinetic energy during TD, the inclusion of higher mass hadronic resonances, the strange baryon excess and the finite timelike thickness of the deflagration front may decrease the temperature after TD and the freeze-out times even more.

A model calculation to confirm the above considerations is presented in Figure 1. The initial state, labelled by $I$ corresponds to the PCM temperature curve [2] given as $T(t)=$ 950. $(0.05 \mathrm{fm} / \mathrm{c} / t)^{0.3} \mathrm{MeV}$. This state is taken as an initial state for a $d=3$ scaling Bjorken expansion with $n_{F}=0$. The entropy ratio, $R$, is below 1 in the beginning, indicating that TD-s are not yet allowed. The new channel for TD-s opens at $t=7.3 \mathrm{fm} / \mathrm{c}$ when the entropy ratio first becomes equal to 1. A sudden TD corresponds to a vertical jump from A to A', or to another vertical jump from any point of the $A B$ curve to the $A^{\prime} B$ (dashed) curve. This curve gives the upper bound for the temperature of the available final states. A lower bound may be also given since no final states are allowed with lower entropy than at A, which yields a Poisson adiabat $A^{\prime} A^{\prime \prime}$. Thus the available final states with $s_{f}$ are given by the hatched A'A"B region since $s_{I}<s_{A} \leq s_{F} \leq s_{B}$.

In Figure 1, part of the hatched region lays below the $T / T_{c}=1$ line which indicates that TD-s from the nucleation scenario to hot (but not superheated) hadronic gas are also possible. These transitions may happen at times close to the end of the nucleation where the initial hadronic fraction is already close to 1.

In summary, we considered the time-scales of rehadronization for a baryon-free QGP at RHIC and LHC energies. The time-scale for reaching the bottom of the temperature curve during the cooling process via homogeneous nucleation [4] is surprisingly close to the time-scale of the freeze out, as estimated based on the analysis and extrapolation of present high energy HBT data. The non-equilibrium nucleation scenario leads to the development of 
mechanical instability of the supercooled QGP phase which then may be suddenly converted in a timelike deflagration from the supercooled state to a (super)heated hadronic matter. In a model simulation such a sudden process was indeed possible and satisfied energy and momentum conservation with non-decreasing entropy. It is possible to reach a hadronic state frozen out immediately after the timelike deflagration. If a TD leads to a simultaneous hadronization and freeze-out, the conditions of a pion-flash are satisfied. This rehadronization mechanism is signalled by a reduced (if not vanishing) difference between the sidewards and outward components of Bose-Einstein correlation functions, in the observation of the free masses of the resonances in the dilepton spectra, and in a clean strangeness signal of the QGP.

Acknowledgments: We thank J. Kapusta, D. Boyanovsky, M. Gyulassy, P. Lévai and G. Wilk for stimulating discussions. This work was supported in part by the Norwegian Research Council (NFR) under Grant No. NFR/NAVF-422.93(94)/008, /011, by the NFR and the Hungarian Academy of Sciences exchange grant, by the Hungarian NSF under Grants No. OTKA-F4019 and OTKA-2973 as well as by the US NSF under Grant No. PHY89-04035 and NATO SAF Grant \# CRG.920322-644/92/JARC-50/. 


\section{REFERENCES}

[1] Proceedings of the Quark Matter conferences, especially Nucl. Phys. A498, (1989), Nucl. Phys. A525, (1991), Nucl. Phys. A544, (1992) and Nucl. Phys. A566, (1993).

[2] K. Geiger and B. Müller, Nucl. Phys. B369, 600 (1992); K. Geiger, Phys. Rev. D 46, 4965, 4986 (1992); ibid. 47 133, (1993).

[3] T. DeGrand and K. Kajantie, Phys. Lett. B147, 273 (1984); and references therein.

[4] L. P. Csernai and J. I. Kapusta, Phys. Rev. Lett. 69, 737 (1992); and Phys. Rev. D 46, 1379 (1992).

[5] L. P. Csernai, et al., Z. Phys. C 58, 453 (1993).

[6] These are defined in the longitudinally comoving system (LCMS) of the boson pair. The longitudinal direction, $L$, in LCMS is parallel to the beam, the out direction, $T$, out, in LCMS is parallel to the total momentum of the pair, and the side direction, $T$, side, is transverse both to the beam and the total momentum of the pair.

[7] T. Csörgő and S. Pratt, KFKI-1991-28/A, p. 75; S. Pratt, Phys. Rev. D 33, 1314 (1986).

[8] A. Makhlin and Y. Sinyukov, Z. Phys. C39, 69 (1988).

[9] J. Bolz, et al., Phys. Lett. B300, 404-409 (1993)

[10] R. Stock, Annalen der Physik 48, 195 (1991);

[11] H. Satz, Nucl. Phys. A544, 371c (1992).

[12] J. Rafelski, Phys. Lett. B262, 333 (1991);

[13] S. Abatzis, et al., WA85 Collaboration, Phys. Lett. B259, 508 (1991); Phys. Lett. B270, 123 (1991);

[14] J. Kapusta \& A. Mekjian, Phys. Rev. D33, (1986) 1304. 
[15] N. Bilić, et al., Phys. Lett. B311 266 (1993); N. Bilić, et al., preprint CERN-TH 6923/93. J. Cleymans, et al., Z. Phys. C 58, 347 (1993).

[16] J. Harris in "Particle Production in Highly Excited Matter", H. Gutbrod and J. Rafelski eds, NATO ASI series, B303 (Plenum, 1993) p. 89

[17] P. Lévai, et al, Proc. XVIII Hirschegg Workshop, Austria, Jan. 1990 (ed. H. Feldmeier) p. 90.

[18] A. K. Holme, et al., Phys. Rev. D40, 3735 (1989).

[19] L.P. Csernai and M. Gong, Phys. Rev. D37, 3231 (1988).

[20] E. V. Shuryak, Phys. Rev. Lett. 70, 2241, (1993); K. Geiger, Nucl. Phys. A566 257c (1994), K. J. Eskola and X.-N. Wang, nucl-th/9307011 


\section{Figure Captions:}

Fig. 1.: Opening of a new channel for a TD from supercooled $n_{F}=0$ Gluonic Plasma to hadron gas. The label $I$ indicates the initial state for a 3D scaling Bjorken expansion for $A u+A u$ collisions at RHIC energy, corresponding to a PCM temperature at $t=3.5 \mathrm{fm} / \mathrm{c}$. The solid curve, $I A A^{\prime \prime} B B^{\prime \prime}$, stands for the nucleation scenario. The critical temperature is reached at $t_{c}=5.6 \mathrm{fm} / \mathrm{c}$. The dotted line, labelled by $R$, indicates the entropy ratio for a sudden TD from the initial state (given by the nucleation scenario) to a hadronic final state. This becomes first possible at A where the system may jump to A'. TD-s from the points of the $A B$ line vertically up to the points of the $A^{\prime} B$ line are possible, since there $R \geq 1$. The available final states are within the hatched area due to entropy constrains. No bubble-fusion was considered, bubbles grew together with the scaling expansion of the matter, and the surface tension was $\sigma=20 \mathrm{MeV} / \mathrm{fm}^{2}$. 


\section{FIGURES}

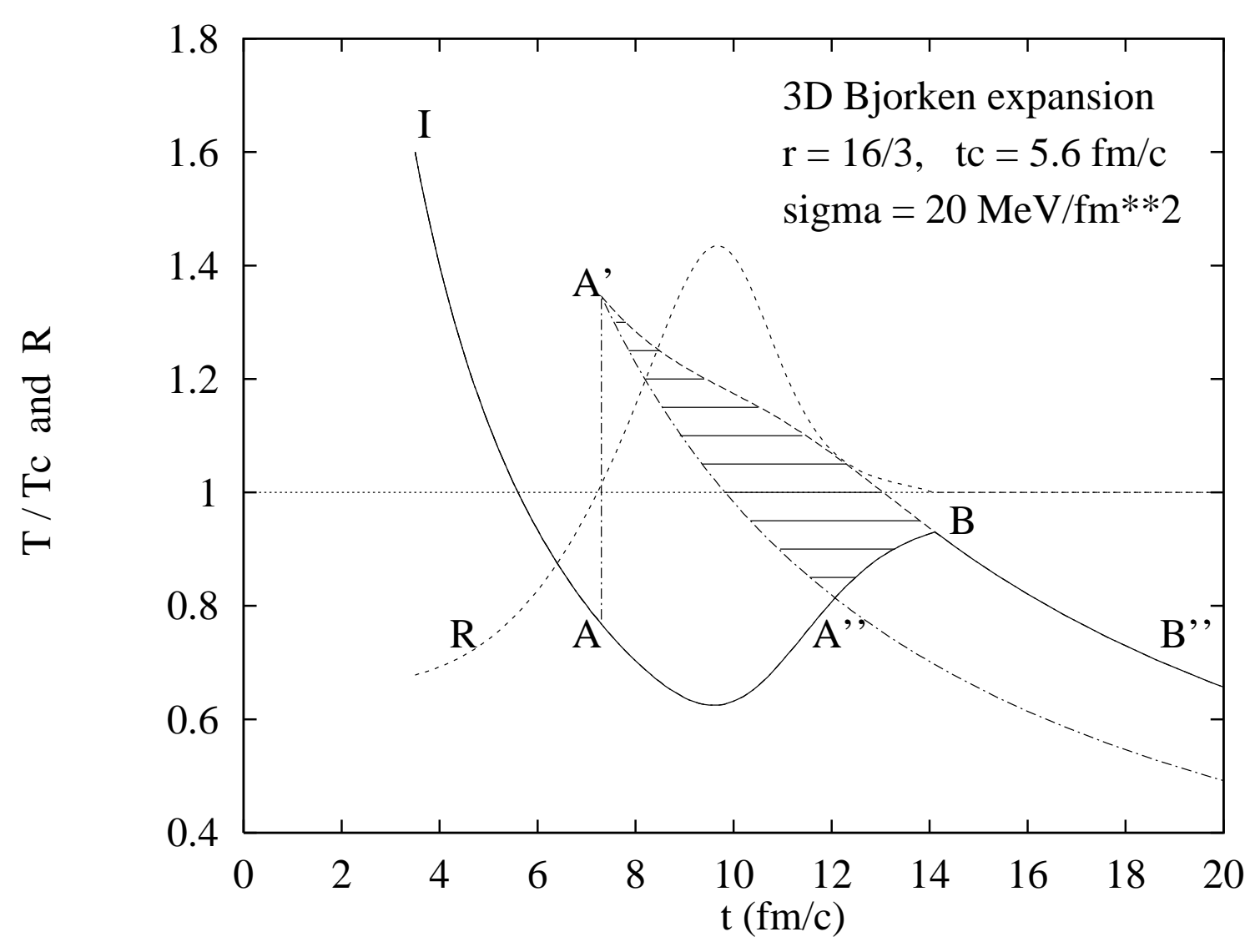

Figure 1. 